\title{
Lingua Franca English, Multilingual Communities, and Language Acquisition
}

\author{
SURESH CANAGARAJAH \\ Pennsylvania State University \\ 304C Sparks Building \\ University Park, PA 16801 \\ Email: asc16@psu.edu
}

\begin{abstract}
Firth and Wagner (1997) questioned the dichotomies nonnative versus native speaker, learner versus user, and interlanguage versus target language, which reflect a bias toward innateness, cognition, and form in language acquisition. Research on lingua franca English (LFE) not only affirms this questioning, but reveals what multilingual communities have known all along: Language learning and use succeed through performance strategies, situational resources, and social negotiations in fluid communicative contexts. Proficiency is therefore practicebased, adaptive, and emergent. These findings compel us to theorize language acquisition as multimodal, multisensory, multilateral, and, therefore, multidimensional. The previously dominant constructs such as form, cognition, and the individual are not ignored; they get redefined as hybrid, fluid, and situated in a more socially embedded, ecologically sensitive, and interactionally open model.
\end{abstract}

The concept of language as a rigid, monolithic structure is false, even if it has proved to be a useful fiction in the development of linguistics. It is the kind of simplification that is necessary at a certain stage of a science, but which can now be replaced by more sophisticated models. (Haugen, 1972, p. 325)

Firth and Wagner questioned some key dichotomies operative in second language acquisition (SLA) research in their 1997 article. Focusing mainly on the constructs learner versus user, nonnative versus native speaker (NNS vs. NS), and interlanguage versus target language, they contested the notions of deficiency imputed to the first construct in each pair. SLA ${ }^{1}$ has generally worked with the assumption that learners are emulating the idealized competence of NSs, that they are handicapped in their capacity to communicate with the undeveloped language they possess, and that learning a language primarily constitutes

The Modern Language Journal, 91, Focus Issue, (2007) 0026-7902/07/923-939 \$1.50/0

(C)2007 The Modern Language Journal mastering its grammar in specially designed pedagogical contexts.

The ensuing debate has made us aware of many other dichotomies in language acquisition that need to be reexamined:

1. Grammar versus pragmatics: Is one more primary in communication than the other, and are they in fact separable? Would pragmatic strategies enable one to communicate successfully irrespective of the level of grammatical proficiency? (House, 2003).

2. Determinism versus agency: Are learners at the mercy of grammar and discourse forms for communication, or do they shape language to suit their purposes? (Canagarajah, 2006a).

3. Individual versus community: Are language learning and use orchestrated primarily by the individual even when they occur through interaction? Or do communication and acquisition take place in collaboration with others, through active negotiation, as an intersubjective practice? (Block, 2003).

4. Purity versus hybridity: Are languages separated from each other, even at the most abstract level of grammatical form? And how do they associate with other symbol systems and modalities of communication? (Khubchandani, 1997; Makoni, 2002). 
5. Fixity versus fluidity: What is the place of deviation, variation, and alteration in language, and can a system lack boundedness? Similarly, is acquisition linear, cumulative, unidirectional, and monodimensional? (Kramsch, 2002; Larsen-Freeman, 2002).

6. Cognition versus context: Do we formulate and store language norms detached from the situations and environment in which they are embedded? Is learning more effective when it takes place separately from the contexts where multiple languages, communicative modalities, and environmental influences are richly at play? (Atkinson, Churchill, Nishino, \& Okada, 2007; Lantolf \& Thorne, 2006).

7. Monolingual versus multilingual acquisition: Should we treat learning as taking place one language at a time, separately for each, in homogeneous environments? (Cook, 1999).

Firth and Wagner (1997) ushered in the questioning of the dichotomies, and we have gradually progressed to a position of model building, developing alternate theoretical paradigms that would integrate these constructs (see Zuengler \& Miller, 2006, for a review). Although Firth and Wagner primarily sought parity between the constructs, we are now in a position not only to abandon the dichotomized orientation but also to synthesize the constructs on a radically different footing. Firth and Wagner ended their article with a broad call "to work towards the evolution of a holistic, bio-social SLA" (p. 296). We have hence/now constructed a range of specific models that elaborate and refine the biosocial paradigm (examples follow). Thus the first of the three requirements Firth and Wagner identified in order to redress the imbalance-in other words, an enhanced awareness of the contextual and interactional dimensions of language use-stands fulfilled. The other two requirements-in other words, an emic perspective and a broadened SLA database-are still to be realized. We need more insider studies from multilingual (especially non-Western) communities and data from outside the classroom to meet these requirements. Even in the case of theoretical awareness and model building, we do not have a consensus. Zuengler and Miller (2006) argued that the cognitive and social perspectives constitute "parallel worlds" in SLA studies (p. 35).

It is in this context that I present recent research related to lingua franca English (LFE) ${ }^{2}$ as radically reconfiguring the new models of language usage and acquisition being constructed in our field. This emergent body of knowledge enables us to reappraise the constructs that were previously ignored or suppressed. To a considerable extent, LFE research presents data from contact situations in professional and everyday contexts outside the classroom, broadening the SLA database. Though we need more emic perspectives from non-Western communities, the studies by European scholars provide useful data from multilingual contexts. LFE research was available earlier, but it has developed to even more complex levels as the global currency of English has grown in relation to recent forms of postmodern globalization. ${ }^{3}$ The new context, featuring transnational affiliations, diaspora communities, digital communication, fluid social boundaries, and the blurring of time-space distinctions has generated more information about atypical communicative contexts, encouraged studies on contact situations, and created an urgency to understand acquisition outside homogeneous communities.

Therefore, we now have new data and perspectives that were unavailable at the time of the initial debate. However, my argument here is not that SLA has to be revised only to accommodate the exceptional issues deriving from globalization and LFE. ${ }^{4}$ These recent developments have only made us aware of some fundamental processes of language learning and usage relevant to diverse communities in different historical and geographical contexts. For example, we are now in a position to appreciate how language learning and usage have taken place in non-Western multilingual communities for centuries. The local knowledge of these periphery communities has been ignored in linguistic scholarship, as in many other fields in the academy.

Therefore, we should consider the critique, revision, and expansion of dominant constructs as a desirable process of knowledge construction. As Haugen (1972) noted, in the quotation in the epigraph, there is a place for enabling fictions at particular stages in scholarly inquiry. However, in the light of new evidence, especially as social conditions themselves change and our inquiry becomes sharper, we have to deconstruct our earlier models and perhaps start anew. Globalization, multilingual contact, and LFE provide impetus for continuing this disciplinary rethinking with new urgency and addressing language processes and practices that have lain hidden all the time.

In this article, I first review studies on the acquisition and use of English as a contact language. Based on these research findings, I consider the 
implications for the dichotomized constructs in SLA. Then, I review the literature on communicative practices in non-Western communities, which confirms the practices informing LFE usage, suggesting the bases of the resources and skills multilinguals bring to language negotiation. The non-Western scholarship also raises additional complex questions about language use and acquisition that enable us to further advance our inquiry on SLA. As I move toward an alternate paradigm, I consider the reasons why such acquisition processes have not been addressed in the dominant SLA models. Examining the (structuralist) philosophical and (monolingual) social biases in knowledge construction, I move on to outline a new integration of the SLA constructs on a practice-based model that would better accommodate the communicative processes of multilinguals.

\section{ACQUIRING AND USING LINGUA FRANCA ENGLISH}

Graddol (1999) prophesied "in [the] future [English] will be a language used mainly in multilingual contexts as a second language and for communication between non-native speakers" (p. 57). This prediction is arguably already a reality. English is used most often as a contact language by speakers of other languages in the new contexts of transnational communication. Speakers of English as an additional language are greater in number than the traditionally understood $\mathrm{NSs}^{5}$ who use English as their sole or primary language of communication. These developments have impressed upon us the need to understand the character of LFE, a variety that overshadows national dialects-the dominant ones such as British or American English and the recently nativized forms such as a Indian or Singaporean English-both in currency and significance (see Canagarajah, 2006b; Jenkins, 2006; and Seidlhofer, 2004, for the state of the art on LFE). How is this lingua franca, ${ }^{6}$ a language so important for millions of global speakers, acquired and used?

LFE belongs to a virtual speech community. The speakers of LFE are not located in one geographical boundary. They inhabit and practice other languages and cultures in their own immediate localities. Despite this linguistic-cultural heterogeneity and spatial disconnect, they recognize LFE as a shared resource. They activate a mutually recognized set of attitudes, forms, and conventions that ensure successful communication in
LFE when they find themselves interacting with each other. House (2003) appropriately called these communities of imagination, borrowing the well-known metaphor from Anderson (1984). It is unclear what constitutes the threshold level of English proficiency required to join this invisible community. Though some proficiency in English is certainly necessary, it is evident that even those individuals with a rudimentary knowledge can conduct successful communication while further developing their proficiency. This facility is no doubt attributable to the language awareness and practices developed in other contexts of communication with local languages. Multilingualism is at the heart of LFE's hybrid community identity and speaker proficiency.

A radical implication of this multilingualism is that all users of LFE have native competence of LFE, just as they have native competence in certain other languages and cultures. This characterization goes against our usual ways of using the concept of NS. Typically, one is an NS of only one language. However, this type of native competence (and insider status) in multiple languages is a well-known reality in many communities. LFE only makes this phenomenon more visible and global. An important implication is that unlike our treatment of those who are outsiders to British, American, or other national varieties of English, we cannot treat LFE speakers as incompetent. House (2003) put it this way: "a lingua franca speaker is not per definitionem not fully competent in the part of his or her linguistic knowledge under study" (p. 557). This assertion does not mean that LFE speakers do not develop their proficiency further-just as Anglo-American NSs still have to develop their proficiency in English. Perhaps we have to distinguish between competence and proficiency. Both LFE speakers and NSs have competence in their respective varieties, though there is no limit to the development of their proficiency through experience and time. The competence of LFE speakers is of course distinct. This competence for cross-language contact and hybrid codes derives from their multilingual life.

Because of the diversity at the heart of this communicative medium, LFE is intersubjectively constructed in each specific context of interaction. The form of this English is negotiated by each set of speakers for their purposes. The speakers are able to monitor each other's language proficiency to determine mutually the appropriate grammar, phonology, lexical range, and pragmatic conventions that would ensure intelligibility. Therefore, it is difficult to describe this language a priori. It 
cannot be characterized outside the specific interaction and speakers in a communicative context. Meierkord (2004) said that LFE "emerges out of and through interaction" and, for that reason, "it might well be that ELF never achieves a stable or even standardized form" (p. 129). In this sense, LFE does not exist as a system "out there." It is constantly brought into being in each context of communication.

Let us now unpack the implications of this negotiability for form. The form of LFE is variable. ${ }^{7}$ Because the type of language is actively negotiated by the participants, what might be inappropriate or unintelligible in one interaction is perfectly understandable in another. This notion of form goes beyond the traditional understanding of variation as deriving from a common core of grammar or language norms. In other words, variation is at the heart of this system, not secondary to a more primary common system of uniform norms. Speakers understand the interlocutor's variants and proceed effectively with the communication, in turn using their own variants. As Gramkow Anderson (1993) put it "there is no consistency in form that goes beyond the participant level, i.e., each combination of interactants seems to negotiate and govern their own variety of lingua franca use in terms of proficiency level, use of code-mixing, degree of pidginization, etc." (p. 108).

To make matters more complicated, LFE's form is hybrid in nature. The language features words, grammatical patterns, and discourse conventions from diverse languages and English varieties that speakers bring to the interaction. Participants borrow from each other freely and adopt the other's language in their interaction with that participant. In her research on the syntactic character of LFE, Meierkord (2004) presented it as a heterogeneous form of English characterized by: (a) "overwhelming correspondence to the rules of L1 Englishes"; (b) "transfer phenomena, developmental patterns and nativised forms"; and (c) "simplification, regularisation and levelling processes" (p. 128).

Sampson and Zhao (2003) made an analogy between LFE and a pidgin language, based on data from multilingual sailors. They found the existence of Singaporean, Indian, and Phillipino Englishes, in addition to other languages, in the LFE of their participants. The sailors borrowed from the usage of each other to develop a hybrid language that is still shared and used smoothly for communication. Thus, LFE raises serious questions about the concept of language system. Is it possible to consider form as constituting an indeterminate, open, and fluid system?

How does such a fluid system facilitate harmonious communication? It is obvious that LFE speakers cannot depend on a preconstituted form for meaning. They activate complex pragmatic strategies that help them negotiate their variable form. It is amazing, therefore, that "misunderstandings are not frequent in ELF interactions," according to Seidlhofer (2004, p. 218). She went on to say that "when they do occur, they tend to be resolved either by topic change, or, less often, by overt negotiation using communication strategies such as rephrasing and repetition" (p. 218). A kind of suspension of expectations regarding norms seems to be in operation, and when forms from a different language or English variety surface, they do not interfere negatively. Planken (2005) described how this condition is achieved in intercultural business communication. She noted that the interlocutors do some preparatory work through opening comments to create a third space-a no-man's-land between their primary languages and cultures-to negotiate LFE on equal terms. Through reflexive comments on their own communicative practices, self-deprecating humor, and the evocation of their shared nonnativeness, they distance themselves from their own norms and activate flexible practices that facilitate communication.

As long as a certain threshold of understanding is obtained, interlocutors seem to adopt what Firth (1996) termed the let it pass principle, by which they overlook idiosyncracies. Part of these pragmatic resources are discourse strategies (at the suprasentential level) to accommodate local variants. Meierkord (2004) found that although individuals retain the characteristics of their own English varieties, they facilitate communication through syntactic strategies like segmentation (involving utterances that are shortened into clausal or phrasal segments that form the basic informational units) and regularization (involving the movement of focused information to the front of the utterance). These characteristics give the impression that LFE talk is "overtly consensusoriented, cooperative and mutually supportive, and thus fairly robust" (Seidlhofer, 2004, p. 218).

If uniformity of form is not a requirement in LFE, more surprising is the finding that even the enabling pragmatic strategies do not have to be the same. House (2003) demonstrated how students of English from different countries 
bring pragmatic strategies valued in their own communities to facilitate communication with outsiders. These strategies are, paradoxically, culture-specific strategies that complement intercultural communication. For example, House found that "Asian participants employ topic management strategies in a striking way, recycling a specific topic regardless of where and how the discourse had developed at any particular point" (p. 567). This discourse of parallel monologues actually helps nonproficient English speakers because it enables them to focus on each move as if it were a fresh topic. In the three strategies House described, while the local cultural ways of interacting are alive in the English of Asians, they still serve to ensure intelligibility and communication with outsiders. This communication is possible because the other also brings his or her own strategies to negotiate these culture-specific conventions. Participants, then, "do their own thing," but still communicate with each other. Not uniformity, but alignment is more important for such communication. Each participant brings his or her own language resources to find a strategic fit with the participants and purpose of a context.

For communication to work across such radical differences, it is important that acquisition and use go hand in hand. As speakers use LFE, a lot of learning takes place: They monitor the form and conventions the other brings; they learn to ascribe meanings to their form and conventions; and they monitor their own form and convention to negotiate communication. Meeting different speakers from the vast, diffuse, and virtual community of LFE, one always has to learn a lot-and rapidlyas one decides which receptive and productive resources to adopt for a context. Furthermore, the lessons learnt in one encounter will help to constantly reconstruct the schema to monitor future communication of similar or different participants and contexts. In this sense, learning never stops in LFE. If there is no language use without learning, there is also no language learning outside of use. Because there is no a priori grammar, the variable language system has to be encountered in actual use. The contexts of intercultural global communication are unpredictable, and the mix of participants and purposes have to be encountered in real situations. Also, the strategies that enable negotiation are meaningless as knowledge or theory; they have to be constantly activated for their development. A language based on negotiation can be developed only through and in practice.

\section{Implications for Theorizing Acquisition}

Such a scenario of LFE communication complicates the dominant constructs of SLA and validates the questions raised by Firth and Wagner (1997). Because LFE is intersubjectively constructed in a situation- and participant-specific manner, it is difficult to elicit a baseline data to assess the proficiency of LFE speakers. LFE's form and conventions vary for different speakers and contexts. We have to judge proficiency, intelligibility, and communicative success in terms of each context and its participants. More importantly, we have to interpret the meaning and significance of the English used from the participants' own perspective, without imposing the researcher's standards or criteria invoked from elsewhere.

A related point here is that we have to rid ourselves of what Cook (1999) called the comparative fallacy. The haste to judge language performance using limited and unfair norms has affected much of what we have done so far in language learning. ${ }^{8}$ The treatment of a putative NS of English as the norm is another manifestation of the comparative fallacy. The English of multilingual LFE speakers is not used in deference to the norms of prestige varieties such as British or American English. LFE speakers do not treat the speakers of these varieties as their frame of reference. House (2003) reminded us "the yardstick for measuring ELF speakers' performance should therefore rather be an 'expert in ELF use', a stable multilingual speaker under comparable sociocultural and historical conditions of use, and with comparable goals for interaction" (p. 573). This is a tongue-in-cheek statement, as we have seen that there is nothing stable about the multilingual speaker. Moreover, there is little that is comparable about LFE contexts or purposes of interaction, as each LFE interaction ushers in its own unique dynamics.

These realizations call into question the idea that the English of multilingual users is an interlanguage. Multilingual speakers are not moving toward someone else's target; they are constructing their own norms. It is meaningless to measure the distance of LFE speakers from the language of Anglo-American speakers as LFE has no relevance to their variety. Besides, we have to question the assumption in the interlanguage concept that there are gradations, a linear progression, and an endpoint to be achieved in language learning. We have seen that each LFE interaction is a unique context, raising its own challenges for negotiation. 
It may not be the case that one communicative act contributes to the other and so on, leading to a cumulative line of progression. Because the contexts are so variable and unpredictable, it is not possible to say that a target can be reached for perfect or competent LFE proficiency. (We may not be able to say that even for Anglo-American NSs of English.)

If at all, we can speak of achieving a type of language awareness and competence that can help handle diverse communicative situations. However, it is possible that multilinguals already come with this competence and do not wait for their interactions in English to develop that ability. Based on her findings of the creative and complex negotiation strategies of multilinguals, House (2003) argued "all these strategies seem to show that ELF users are competent enough to be able to monitor each others' moves at a high level of awareness" (p. 559). In this sense, their development of LFE proficiency has to be granted relatively greater agency, at least analogous to the agency attributed to the development in one's first language in certain generativist models. The LFE speaker comes with the competence-in many respects, more advanced than that of the child because of the years of multilingual practice enjoyed in their local communities-which is then honed through actual interactions. This development does not have to be marked by miscommunication or deficient usage, and should not be treated as such.

We realize, however, that the linguistic competence of an LFE speaker has to be defined more broadly and with greater complexity. The dominant orientation is to treat solely or mainly form as defining competence, with communicative competence given a secondary role. In LFE, form receives reduced significance; or, rather, form gets shaped according to the contexts and participants in an interaction. More important are a range of other skills, abilities, and awareness that enable multilingual speakers to negotiate grammar. In addition to grammatical competence, we have to give equal importance to: language awareness that enables speakers to make instantaneous inferences about the norms and conventions of their multilingual interlocutors; strategic competence to negotiate interpersonal relationships effectively; and pragmatic competence to adopt communicative conventions that are appropriate for the interlocutor, purpose, and situation. $^{9}$

The orientation to acquisition as a cognitive activity also needs clarification. We cannot focus on the activity and the content of the mind in understanding LFE proficiency. There is a considerable contribution from environmental and social domains. The rules, schema, and conventions developed by LFE users come loaded with significant social information. The variable and hybrid grammar of LFE cannot be acquired outside the contexts and social milieu that help select them and give meaning. If language has a cognitive habitation, such a cognition is shaped, enabled, and realized in social practice. In this respect, the distinction between competence and performance has to be revised. It can be argued that in the case of LFE, there is no meaning for form, grammar, or language ability outside the realm of practice. LFE is not a product located in the mind of the speaker; it is a form of social action.

In theorizing this complex social action, some scholars have begun to explore how successful communication depends on aligning the linguistic resources one brings to the social, situational, and affective dimensions operative in a context (see Kramsch, 2002). In other words, language learning involves an alignment of one's language resources to the needs of a situation, rather than reaching a target level of competence. Atkinson et al. (2007) defined alignment as "the means by which human actors dynamically adapt to-that is, flexibly depend on, integrate with, and construct-the ever-changing mindbody-world environments posited by sociocognitive theory. In other words, alignment takes place not just between human beings, but also between human beings and their social and physical environments" (p. 171, original emphasis). Atkinson and his collaborators went on to illustrate alignment through the English language learning interaction of a Japanese child and her tutor. What is more pertinent to this article (an issue the authors do not choose to develop) is the way both Japanese and English and, sometimes, coconstructed words and meanings of ambiguous linguistic identity are used as cues and effects of successful alignment to facilitate English language learning.

This notion of alignment makes us question another bias in SLA-language acquisition as an individual activity. It is clear that the individual's proficiency is shaped by collective and contextual factors. But there are other implications for assessing an individual's level of proficiency. As we saw, LFE makes sense only as an intersubjective construction, something achieved by two or more people, based on the strategies they bring to the interaction. We have to consider the collaborative nature of communication and linguistic negotiation in assessing the meaning and significance of an interaction. 
From this perspective, the conduit model of meaning as information transfer (which informs SLA) has to be questioned. In LFE, meaning does not precede (and is not detachable from) the language in which it is communicated. House (2003) noted, "in ELF use, speakers must continuously work out a joint basis for their interactions, locally construing and intersubjectively ratifying meanings" (p. 559). Therefore, even an ungrammatical usage or inappropriate word choice can be socially functional. They can create a new meaning originally unintended by the speaker, or they may be negotiated by the participants and given new meanings. Participants negotiate the language effectively to ascribe meaning to everything. A radical implication of this assertion for assessing language proficiency is that error is also socially constructed. An error occurs when someone fails to ascribe meaning to a linguistic form used by another. In LFE, such cases rarely occur. Breakdown in LFE communication is possible only in rare cases of refusal to negotiate meanings-which is itself a form of communication as it conveys the participant's desire to cut off the conversation. Therefore, if there is a case of failed communication, we cannot blame an individual for lack of proficiency. This failed communication might be a divergence strategy (Giles, 1984). Those individuals who assess proficiency have to take into account such joint activity of participants in communication before rushing to rule something a mistake.

In relation to all these issues, we have to question whether researchers can study language acquisition by standing outside the interaction in question or, even worse, coming from outside the communities they study. Would they be imposing norms and meanings that do not matter to their participants? Given the intersubjective nature of LFE, how can researchers who do not participate in a specific communicative event claim to be privy to the norms and meanings operative for those involved? There is research documentation to suggest that in cases where speakers do not come from the LFE virtual community, sharing the basic communicative expectations, their interaction fails (House, 2003).

Ironically, the only cases of miscommunication House (2003) observed in her research were in the interactions of multilingual speakers with those individuals for whom English is native or sole language. This miscommunication in nativenonnative talk is easy to explain, as NSs would fail to negotiate, treating their norms as universally applicable. Would researchers be prone to similar misunderstanding, especially in cases where they are NSs, in addition to being outsiders to the interaction?

The intersubjective nature of communication makes us question the separation of the learner role from other social roles and identities. In traditional SLA research, a learner's language is not presumed to be functional (unless proven otherwise). The researcher's acts of othering, objectifying, patronizing, and judging further reduce the learner's social complexity. However, LFE users are always conscious of the social roles they play in their contexts of contact communication. We have to interpret their performance in terms of the purposes and roles that matter in that speech event. To further complicate theorization, LFE users do not remain with the rich and diverse identities they bring to the event; as we discussed earlier, they negotiate to modify and reconstruct new identities more amenable to the interaction. Therefore, to reduce the analysis to speaker-aslearner is to leave out many other features of communication that provide significance to the language data.

This recognition does not mean that other social identities may not subsume the learner identity, or vice versa. We now know that in all language learning contexts, including academic venues, acquisition is a social process where subtle nonpedagogical meanings and identities are communicated. Even in classroom contexts, identities are multiple, conflictual, and changing (see Norton, 2000). Students convey other meanings and identities not prescribed in the lessons.

In my ethnography of classroom discourse, I show how students shuttle between identities of learner, friend, and in-group community member with their teachers, all the time conveying contextually relevant meanings, even as they gain communicative competence in codeswitching (Canagarajah, 1999). They also find spaces for expressing resistant identities, deviating from the institutionally mandated roles and distancing themselves from messages of the hidden curriculum. Often even narrowly defined pedagogical exercises can be turned into richly purposive communication by students. Learners can subvert lessons that treat them as passive and mechanical through sarcasm, serving to prove themselves complex agents. Routine pedagogical exercises can be reframed to generate humour and play. These communicative acts and identities can imply complex proficiency. By the same token, interactions that are not framed as pedagogical (i.e., off-task, off-site activities) can be utilized for learning. These realizations make us question the assumption of learning as a conscious, controlled, 
predesigned, and predictable activity. We have to move toward conceiving of learning as often nonintentional, nonscripted, and nonlinear to understand LFE acquisition in everyday contexts.

As we consider acquisition as transcending the control of the individual and the scope of interpersonal relationships, we have to explore one's language development in relation to that of a whole community of speakers. When a language is being appropriated by a community to suit its own interests and values, developing unique grammars and conventions in the process, should we still assess the language of the individual in relation to NS norms? The term macro-acquisition has been used to understand how a community appropriates another language and develops proficiency in endonormative terms (Brutt-Griffler, 2002). We have to develop ways to map the microacquisition of individuals with the macroacquisition of the communities of which they are a part. This process is not always isomorphic. In some ways, the individual has to align his or her learning to that of the community's norms; in other ways, he or she has to deviate from and resist the norms of the collective for the sake of voice and individuality. To make matters more complicated, while mapping the levels of alignment of the individual and the collective, we also have to realize that the language development of both is mobile and changing. In this sometimes asynchronous proficiency development in an unstable grammatical system, one needs creative strategies to make the appropriate alignment between one's language resources and the requirements of the context.

We can now appreciate how certain methodological constructs that were a cause of concern for Firth and Wagner (1997) are constitutive features of LFE. These features are the need to (a) consider meaning as negotiated and intersubjective; (b) treat form as shaped by participants for their own purposes in each communicative activity; (c) affirm learners as capable of exerting their agency to renegotiate and overcome errors; (d) integrate learning and use; (e) provide for nonlearner social identities in acquisition; (f) accommodate both purposive everyday communication and nonfunctional play as equally contributing to acquisition; (g) relate to language as practice; (h) treat cognition as situated and competence as performance; and (i) interpret the communication of novices in context without comparing it with NS norms or a target proficiency, or treating it as an interlanguage. The use of these methodological constructs is not optional, as Firth and Wagner seemed to allow, for different modes and cases of analysis. We cannot choose to either adopt or ig- nore them in the study of LFE acquisition and use. LFE is meaningless outside these conditions.

\section{LANGUAGE ACQUISITION AND USE IN MULTILINGUAL COMMUNITIES}

If multilingual speakers display such stupendous competence in acquiring and using a hybrid language like LFE, there is evidence that it comes from language socialization and awareness developed in their local communities. Higgins (2003) found in her group experiment that multilingual students were more successful in decoding the meaning of lexical and grammatical items from new Englishes than Anglo-American students. NSs had difficulties in such tasks as they did not bring skills and attitudes open to negotiation. The practices we observe in LFE users are common in other contexts of multilingual communication involving local languages. Paradoxically, then, recent findings in LFE communicative practices help us appreciate language acquisition and use common to multilingual communities from precolonial times.

Though we do not have adequate scholarly descriptions of them in our field, these practices are not completely lost in these communities. We are beginning to see descriptions of such practices from Africa (Makoni, 2002), South America (de Souza, 2002), and the Polynesian Islands (Dorian, 2004), among others. They are striking in their differences from the dominant constructs in linguistics, and raise further questions that need exploration. I base the description of multilingual communication that follows on my own region of early socialization in South Asia, especially as it emerges through the perceptive discussion of Khubchandani (1997). ${ }^{10}$

Linguistic diversity is at the heart of multilingual communities. There is constant interaction between language groups, and they overlap, interpenetrate, and mesh in fascinating ways. Not only do people have multiple memberships, but they also hold in tension their affiliation with local and global language groups as the situation demands. Khubchandani (1997) used an indigenous metaphor, Kshetra, to capture this sense of community. Kshetras "can be visualized as a rainbow; here different dimensions interflow symbiotically into one another, responsive to differences of density as in an osmosis" (p. 84). Khubchandani called the unity that develops out of this diversity and continuity of affiliations a superconsensus.

Such individuals and communities are so radically multilingual that it is difficult to identify one's mother tongue or native language. 
People develop simultaneous childhood multilingualism, making it difficult to say which language comes first. As Kubhchandani (1997) pointed out, "identification through a particular language label is very much a matter of individual social awareness" (p. 173). Language identity is relative to the communities and languages one considers salient in different contexts. Therefore, the label is applied in a shifting and inconsistent manner.

Because of such intense contact, languages themselves are influenced by each other, losing their purity and separateness. Many local languages serve as contact languages, and develop features suitable for such purposes-that is, hybridity of grammar and variability of form. Khubchandani (1997) said "many Indian languages belonging to different families show parallel trends of development... [They] exhibit many phonological, grammatical and lexical similarities and are greatly susceptible to borrowing from the languages of contact" (p. 80). He went on to say that differences "between Punjabi and Hindi, Urdu and Hindi, Dogri and Punjabi, and Konkani and Marathi can be explained only through a pluralistic view of language" (p. 91). Though he did not elaborate, the pluralistic view of language would raise many enigmas for traditional linguistics: How do we classify and label languages when there is such mixing? How do we describe languages without treating them as self-contained systems? How do we define the system of a language without the autonomy, closure, and tightness that would preclude openness to other languages?

Such communities are so multilingual that in a specific speech situation one might see the mixing of diverse languages, literacies, and discourses. It might be difficult to categorize the interaction as belonging to a single language. Khubchandani (1997) explained "the edifice of linguistic plurality in the Indian subcontinent is traditionally based upon the complementary use of more than one language and more than one writing system for the same language in one "space" (p. 96; original emphasis). If social spaces feature complementary-not exclusive-use of languages, mixing of languages and literacies in each situation is the norm, not the exception. This communicative reality raises many questions for language acquisition: What kind of competence do people need to communicate in such contexts where different languages mix, mesh, and complement each other? How do people produce meaning out of this seeming chaos of multiple systems of communication?

It is clear that this linguistic pluralism has to be negotiated actively to construct meaning. In these communities, meaning and intelligibility are intersubjective. The participants in an interaction produce meaning and accomplish their communicative objectives in relation to their purposes and interests. In this sense, meaning is socially constructed, not preexisting. Meaning does not reside in the language; it is produced in practice. As a result, "individuals in such societies acquire more synergy (i.e., putting forth one's own efforts) and serendipity (i.e., accepting the other on his or her own terms, being open to unexpectedness), and develop positive attitudes to variations in speech (to the extent of even appropriating deviations as the norm in the lingua franca), in the process of 'coming out' from their own languagecodes to a neutral ground" (Khubchandani, 1997, p. 94, original emphasis).

This description sounds similar to Firth's (1996) let it pass principle and Planken's (2005) noman's-land where participants accommodate differences in language and conventions. Of course, it takes a lot of work to get to this point. Synergy captures the creative agency participants must exert in order to work jointly with the other participant to accomplish intersubjective meaning. Serendipity involves an attitudinal transformation. To accept deviations as the norm, one must display positive attitudes to variation and be open to unexpectedness. Participants have to be radically other-centered. They have to be imaginative and alert to make on-the-spot decisions in relation to the forms and conventions employed by the other. It is clear that communication in multilingual communities involves a different mind-set and practices from the mind-set and practices in monolingual communities.

\section{Implications for Theorizing Acquisition}

How do local people develop proficiency in a form of communication that involves multiple communities and languages in contexts that can generate an unpredictable mix of forms and conventions? How is harmony achieved out of diversity, synchrony out of differences in form and conventions, alignment in discordant and unpredictable situations? Clearly, communication in contact situations is marked by enigmatic paradoxes.

Multilingual communication works because competence does not constitute a form of knowledge, but rather, encompasses interaction strategies. Khubchandani (1997) argued that the ability to communicate is not helped by explicit formulas such as formal grammars and dictionaries of words. For South Asians, "interpretation [is] 
dependent on the focus of communication 'field' and the degrees of individual's 'sensitivity' towards it" (p. 40). In other words, participants must engage with the social context, and responsively orchestrate the contextual cues for alignment. As we have already seen, meaning in language is not a product that can be prescribed objectively. Communication is intersubjective. Rather than knowledge of form, multilingual competence features an array of interactional strategies that can create meaning out of shifting contexts.

As Khubchandani (1997) explained, "communications in everyday life are based on the synergic relationship between the twin criteria: (a) the reciprocity of language skills among communicators (spread over a speech spectrum comprising one or more languages, dialects, styles, etc.); and (b) the mutuality of focus (that is, sharing the relevance of the setting, commonly attributed to the attitudes, moods, or feelings of the participants)" (p. 49). What Khubchandani highlighted are skills and strategies. Mutuality and reciprocity indicate the ways participants align their moves and strategies in relation to their language resources. Synergy is the outcome of this alignment, when participants jointly invoke language resources and collaboratively build coherence. Multilingual competence is thus a mode of practice, not resident solely in cognition.

Furthermore, multilingual competence is open to unpredictability. In a sense, each context of communication poses a new and unpredictable mix of languages and conventions. As Khubchandani (1997) explained further, "it is often difficult to determine whether a particular discourse belongs to language A or B" (p. 93). Therefore, it is difficult to transfer the forms and conventions of one context to the next. In this sense, learning is nonlinear. It is for this reason that when SLA is able to theorize language use and acquisition as based on directed effort (something predictable, with learners armed with a stock of forms and strategies that can make them competent for successful communication), in the Indian community speech is "an effortless integral activity; discourse centres around the 'event' with the support of ad hoc 'expression' strategies" (p. 40). Local people realize that "the 'tradition inspired' standardized nuances of another language or culture" (p. 93) cannot help them communicate successfully in the mix of languages and dialects they encounter in each situation.

It appears as if all that speakers can do is to find a fit-an alignment-between the linguistic resources they bring and the context of communication. Thus, acquisition is not a cumulative process, but an ability to come up with diverse strategies for speech events that need to be addressed for their own sake. The mention of ad hoc strategies reminds us that competence is not predictability but alertness and impromptu fabrication of forms and conventions to establish alignment in each situation of communication. Thus, acquisition aims towards versatility and agility, not mastery and control. ${ }^{11}$

In multilingual competence, grammar receives reduced significance. In contexts where deviation is the norm, multilinguals cannot rely on grammar or form. The linguistics system is a hybrid and variable one, even if it can be described a priori. To reduce further the importance of grammar, Khubchandani (1997) said that the speech process is "regarded as a non-autonomous device, communicating in symphony with other nonlinguistic devices; its full significance can be explicated only from the imperatives of context and communicative tasks" (p. 40). In other words, communication is multimodal. Meaning does not reside in language alone. Linguistic meaning is created in relation to diverse symbol systems (icons, space, color, gesture, or other representational systems) and modalities of communication (writing, sound, visuals, touch, and body), not to speak of diverse languages. If we need a grammar or rules for this mode of communication, it will be a grammar of multimodality-that is, it will contain rules that account for how language meshes with diverse symbol systems, modalities of communication, and ecological resources to create meaning. This orientation would set us on a different path of description from the structuralist tradition that proceeds further inwards into autonomous language to find the rules of linguistic meaning making.

This kind of expanded competence involves not just the rational faculty but other sensory dimensions as well. Kubhchandani (1997) evoked Hindu spiritual concepts to capture this idea: "ancient Indian grammarians talk about the guna (power, potency) of language when deliberating on the dhvani doctrine in Indian aesthetics. A message can convey meaning not merely through its intent in isolation (as indexed in the dictionary) but also in the context of identity (as when observing verbal protocol in a formal setting) or through its effect on the participants (as manipulated by observers)" (p. 52). It is difficult for nonparticipants observing an interaction in a detached manner to come up with a rationalist account for the success of communication. The meaning 
created by the participants, in relation to the dynamics governing that specific interaction, will not be available to outsiders. Because meaning is intersubjective, we have to accommodate the participants' physiological, biological, psychological, affective, and perceptual dimensions in meaningmaking. Khubchandani (1997) warned that "a seemingly incoherent manifestation in these societies can make sense, coalescing into a persuasive whole, almost in spite of disparate elements" (p. 94). A competence for such communication is therefore not only dependent on rational processes, but also involves other dimensions of human subjectivity, requiring a multiscalar mapping of acquisition (see Kramsch, 2002).

Such a competence is always in a state of becoming and, therefore, acquisition is emergent. There is no end point to learning, where one can say a person has mastered all the modalities and dimensions that shape communication in the diverse contact situations. First of all, there is no limit to the diversity, hybridity, and variability that can characterize a language. Furthermore, each interaction, with its own set of participants, interests, and dynamics features new requirements of form and convention. As a result, multilingual competence is treated as always evolving and creative. Khubchandani (1997) explained that the "total verbal repertoire is malleable, responsive to contextual expediencies resulting in uninhibited convergences between speech varieties with the contact pressures of pidginization, hybridization, code-switching and so on" (pp. 40-41). In other words, one's competence is based on the repertoire that grows as the contexts of interaction increase.

In this form of acquisition, therefore, it does not make sense to compare proficiency with baseline data (which would be hard to find as the interactions are so infinitely diverse and unpredictable). We cannot speak of a target to be achieved when the speaker would be perfectly competent for communication at various levels of ability in different contexts. It is also meaningless to speak of interlanguage because competence involves multiple languages with multilateral movement across each of them. A crucial difference here is that SLA accounts for multilingual competence one language at a time, when in reality, this type of competence is more than the sum of the parts, and constitutes a qualitatively different whole.

How does language competence develop out of variable forms, conventions, and modalities of communication? How is competence formed through shifting and unpredictable events? How is order created out of randomness? Can randomness facilitate rational processing and construct patterns of rules and formulae for communication? At the least, we have to think of competence as finding equilibrium between different modalities, hierarchies, and dimensions of communication. Applied linguists have started theorizing such a possibility through chaoscomplexity theory (Larsen-Freeman, 2002), activity theory (Lantolf \& Thorne, 2006), and phenomenology (Kramsch, 2002). In general, they treat competence as an adaptive response of finding equilibrium, rather than a cognitive mastery of rational control. In these orientations, cognition works in context, in situ. Competence is not applying mental rules to situations, but aligning one's resources with situational demands and shaping the environment to match the language resources one brings.

It is for these reasons that multilingual competence cannot rely solely on schools for its development. Because participants have to adopt communicative strategies relevant to each situation and one cannot predict the mix of languages and participants in each context, learning is more meaningful in actual contexts of language use and practice. It is not surprising that, in multilingual communities, language acquisition takes place most effectively in everyday contexts: "In heterogeneous plural environments, a child acquires language from everyday life situations where speech behavior is guided by implicit pressures based on close group, regional, supraregional, and out group identities" (Khubchandani, 1997 , p. 171). It is intriguing how multilingual acquisition has taken place successfully for centuries outside formal schooling in these communities. The multilingual speaker engages with the shifting and fluid situations in everyday life to learn strategies of negotiation and adaptation for meaning-making. Considerable personal appropriation of forms and conventions takes place as the speaker develops skills and awareness that contribute to his or her repertoire-a learning that is ongoing. In sum, acquisition is social practice, not separable mastery of knowledge, cognition, or form.

This description of multilingual competence and acquisition sounds similar in many respects to LFE competence and acquisition. In both, competence is situational, intersubjective, and pragmatic. In both, acquisition is adaptive, practicebased, and emergent. However, we also see some features of acquisition that emerge more distinctly from non-Western scholarship: Multilingual acquisition is nonlinear (i.e., multilateral), noncumulative (i.e., asynchronous), multimodal, 
multisensory, and, therefore, multidimensional. LFE scholars have to consider how their participants might be influenced by such characteristics as they continue their research. Yet, it is fascinating how two research traditions that are not in conversation with each other-and that relate to different geographical locations and historical periods-can come up with such similar descriptions of use and acquisition. If the established knowledge in SLA is not informed by the conditions that characterize language practices that are so pervasive among millions of people in the world, it is important to examine the rationale behind knowledge construction in our field.

\section{RECONSTRUCTING DISCIPLINARY PARADIGMS}

It is now well recognized that the dominant constructs in SLA are founded on monolingual norms and practices. We are also beginning to see a realization among mainstream scholars that these constructs are misleading and distorting (see Dorian, 2004, for a discussion). McLaughlin (McLaughlin \& Sall, 2001) recounted the belated recognition during her field work in Senegal that a local collaborator, whom she discounted as an informant of a language because he was associated with another language, was in fact a proficient insider with authoritative knowledge. Her unitary assumption of the NS did not let her accept her informant as having native proficiency in more than one language. Similarly, Makoni (2002) described how colonial practices of classifying and labeling languages distorted the hybrid reality of South African languages.

These limitations derive from the dominant assumptions of linguistics, informed by the modernist philosophical movement and intellectual culture in which they developed. To begin with, the field treats language as a thing in itself, as an objective, identifiable product. The field also gives importance to form, treating language as a tightly knit structure, neglecting other processes and practices that always accompany communication. Scholars have traced this development to Saussurean linguistics and the structuralist movement (Lantolf \& Thorne, 2006). Other biases follow from this assumption. Khubchandani (1997) pointed out the inordinate emphasis on the temporal life of language, which motivates linguists to chart the linear stages by which imperfect forms develop to a stasis, at which point they become full-fledged forms. Inadequate attention is paid to the way in which various language forms and varieties are embedded in diverse environments, perfectly adequate in their own way for the functions at hand.

Therefore, Kubchandani (1997) called for a spatial orientation. Such an approach would also rectify the lack of attention to the ecological factors of language. We have to understand how language is meshed with other symbolic systems and embedded in specific environments, both shaping and being shaped by them. Mainstream linguistics also fails to give importance to attitudinal, psychological, and perceptual factors that mold the intersubjective processes of communication. This failing is partly due to the primacy of cognition and reason in communication within the mainstream paradigm. There is also a resulting lack of appreciation of the complexity of human communication, which is marked by indeterminacy, multimodality, and heterogeneity. Mainstream linguistics prioritizes the homogeneity of community, competence, and language structure, treating it as the basic requirement that facilitates communication. Even when diversity is addressed, it is treated as a variation deriving from a common form or shared norms.

Critical scholars have discussed the motivations in promoting values based on homogeneity, uniformity, and autonomy in linguistic sciences. They have pointed out how there has been an ideological bias in European history toward unifying communities and identities around a single language (Singh, 1998), treating multilingualism as a problem (Ruiz, 1984), and establishing nationstates around the language of a dominant community (May, 2001). These values are informed by the social conditions and ideologies gaining dominance in Europe since the rise of the nationstate, the 17th-century enlightenment, and the French Revolution (Dorian, 2004; May, 2001). As Dorian (2004) reminded us, "monolingualism, now usually considered the unmarked condition by members of the dominant linguistic group in modern nation-states, was in all likelihood less prevalent before the rise of the nation-state gave special sanction to it" (p. 438). Pratt (1987) interpreted the imposition of homogeneity and uniformity in language and speech community as signifying the construction of linguistic utopias that serve partisan interests. Constructs based on monolingualism and homogeneity are well suited to communities that desire purity, exclusivity, and domination. Acknowledging the heterogeneity of language and communication would force us to develop more democratic and egalitarian models of community and communication. Enabled by such historical processes as colonization and modernity, linguistics has reproduced its 
underlying enlightenment values elsewhere and hindered the development of local language practices and knowledge. ${ }^{12}$

However, in the context of postmodern globalization, as all communities are becoming increasingly multilingual with the transnational flow of people, ideas, and things, scholars are beginning to question the dominant constructs in the field. ${ }^{13}$ Even Western communities are beginning to acknowledge the diversity, hybridity, and fluidity at the heart of language and identity. The struggle now is to find new metaphors and constructs that would capture multilingual communication. How do we practice a linguistics that treats human agency, diversity, indeterminacy, and multimodality as the norm? Because the constructs of modern linguistics are influenced by the modernist philosophical assumptions, some scholars are exploring alternate philosophical traditions to conceptualize these emerging realizations. Phenomenology (Kramsch, 2002), ecological models (Hornberger, 2003), chaos-complexity theory (Larsen-Freeman, 2002), sociocognitive theory (Atkinson et al., 2007), and Vygotskyan sociocultural theory (Lantolf \& Thorne, 2006) are such attempts.

Scholars from postcolonial and non-Western communities are also beginning to represent their communicative practices in scholarly literature from the evidence they still find about them in their communities. This articulation is of course influenced by a worldview and culture that differ from modernity. As we saw in the previous section, Khubchandani (1997) resorted to Indian spirituality and philosophy to represent what he perceives as indigenous language practices. $\mathrm{He}$ uses metaphors like rainbow, symbiosis, osmosis, synergy, and serendipity to describe a multilingual reality that lacks a suitable language in mainstream linguistics. Though these less known publications of periphery scholars are full of insight, they still lack elaborate theorization to produce sophisticated alternative models.

There are other difficulties in working from untheorized local knowledge. One has to break the dominant hermeneutic molds offered by modernism in order to interpret this knowledge. Modernism has denigrated local knowledge, and has interpreted it negatively. Furthermore, local knowledge is not pure or whole, as dominant knowledge systems have appropriated it for their own interests and purposes. At any rate, we must not glorify non-Western traditions. The local can contain chauvinistic tendencies, especially because the onslaught of modernity has been forcing the local to retreat ever further into more recalcitrant positions, in a desperate attempt to maintain its independence. Also, although there are certain egalitarian practices at one level, inequalities in terms of caste, clan, and gender have to be negotiated at other levels of communication. ${ }^{14}$

My effort in this article is not to pit the views of non-Western scholars against emerging models in the West. My proposal is that the insights from non-Western communities should inform the current efforts for alternate theory building in our field. I provide an outline here of how a practicebased model would accommodate the realizations of LFE and multilingual competence. I see this orientation as accommodating the insights of the other model building activities referred to earlier, although going radically beyond the cognitionsociety, or form-pragmatics, dichotomy to integrate them at the level of practice. Though this orientation is informed by the practices of everyday language use and acquisition in non-Western communities, it is also being theorized in the academy by models such as communities of practice (Lave \& Wenger, 1991; Wenger, 1998) and contact zones (Pratt, 1991). ${ }^{15}$

This practice-based model is characterized by the following beliefs and assumptions:

1. What brings people together in communities is not what they share-language, discourse, or values-but interests to be accomplished.

2. These mutual interests would permit individuals to move in and out of multiple communities to accomplish their goals, without considering prior traits that are innate or that are exclusively shared with others.

3. This view would redefine communities as lacking boundedness and a center; they are, rather, contact zones where people from diverse backgrounds meet (Pratt, 1991).

4. What enables them to work together on their interests are negotiation practices they bring to various tasks (not common language, discourse, or values).

5. What enables them to develop expertise in the workings of each community is also practicethat is, engaging actively in purposive activities of that community (not accumulating knowledge and information theoretically without involvement), and acquiring a repertoire of strategies (not information, rules, or cognitive schemata).

6. Identities would then be based on affiliation and expertise rather than those ascribed by birth, family, race, or blood (Rampton, 1990).

7. Though language and discourse enable communication, they are shaped by the practice 
of diverse situations and participants. Form is reconstructed ceaselessly to suit the interests of the participants, in the manner of emergent grammar (Hopper, 1987).

The focus on practice does not mean that there is not a place for classroom learning. Pedagogy can be refashioned to accommodate the modes of communication and acquisition seen outside the classroom (see Canagarajah, 2005a, for a more elaborate pedagogical discussion). Rather than focusing on a single language or dialect as the target of learning, teachers have to develop in students a readiness to engage with a repertoire of codes in transnational contact situations. Although enabling students to join a new speech community was the objective of traditional pedagogy, we now have to train students to shuttle between communities by negotiating the relevant codes. To this end, we have to focus more on communicative strategies, rather than on forms of communication. Students would develop language awareness (to cope with the multiple languages and emergent grammars of contact situations), rather than focusing only on mastering the grammar rules of a single variety. In a context of plural forms and conventions, it is important for students to be sensitive to the relativity of norms. Therefore, students have to understand communication as performative, not just constitutive. That is, going beyond the notion of just constructing prefabricated meanings through words, they will consider shaping meaning in actual interactions and even reconstructing the rules and conventions to represent their interests, values, and identities. In other words, it is not what we know as much as the versatility with which we can do things with language that defines proficiency. Pedagogical movements such as learner strategy training and language awareness go some way toward facilitating such instructional strategies.

These changing pedagogical priorities suggest that assessment too must go through significant changes to evaluate one's ability to negotiate the complex communicative needs of multilingual and contact situations. As we realize that norms are heterogeneous, variable, changing, and, therefore, interactively established in each context, we have to move away from a reliance on discrete-item tests on formal grammatical competence and develop instruments that are more sensitive to performance and pragmatics. Assessment would focus on one's strategies of negotiation, situated performance, communicative repertoire, and language awareness. To this end, we must develop new instruments with imagination and creativity, as I have illustrated it elsewhere (Canagarajah, 2006c).

\section{CONCLUSION}

Although Firth and Wagner (1997) argued for rectifying the imbalance between the dichotomies that characterize SLA, we are now moving toward more radical options of reframing the constructs. The previously ignored or suppressed constructs are now becoming the basis for a new integration or synthesis. Language acquisition is based on performance strategies, purposive uses of the language, and interpersonal negotiations in fluid communicative contexts. The previously dominant constructs such as form, cognition, and the individual are not eradicated; they get redefined to adopt hybrid, variable, situational, and processual characteristics they did not have before. They are treated in a more socially embedded, interactionally open, and ecologically situated manner. The aim of this article is to integrate the dichotomies on the basis of practice, not to reverse the status of competing constructs. Thus, recent research on LFE communication and nonWestern language practices enables us to move the questions raised by Firth and Wagner further along to another level.

Much against the position of some in the original debate that issues of acquisition are separate from broader issues of language communication (see Gass, 1998; Kasper, 1997; Long, 1997), we find that our definitions of language, communication, and communities shape our understanding of acquisition. There are both ideological and methodological implications behind the reexamination of SLA. To return to Haugen (1972), it is true that we work with simple and convenient models of language and acquisition at early stages of knowledge formation. However, models are also interested. That is, they are informed by specific social conditions and their dominant ideologies, and reflect the ends desired by dominant communities. As historical conditions change, and when we encounter new realities, brought to light partly by the critique of existing models, we must construct new paradigms informed by our new knowledge. It is time to revise, reformulate, and refine our models of acquisition for the more egalitarian context of transnational relations and multilingual communication.

\section{NOTES}

${ }^{1}$ Though I relate this discussion to the field of SLA, the article argues that the acquisition I have in mind 
goes beyond the first-second language distinction. Not only is language acquisition always multilingual, it also reveals processes that are similar for languages learnt earlier or later.

${ }^{2}$ Other scholars (e.g., House, 2003; Seidlhofer, 2004) use the acronym ELF (English as a lingua franca). I have retained their acronym when I quote these scholars.

${ }^{3}$ Perhaps Rampton (1997), among all the discussants, anticipated this development best as he outlined how globalization and diaspora life were transforming communication. Ironically, though Firth (1996) was one of the earliest researchers to initiate a study of LFE, he did not couch his argument on SLA in terms of this inquiry. In hindsight, it is possible to guess that it is Firth's LFE research that enabled Firth and Wagner (1997) to pose their questions with such force and foresight.

${ }^{4}$ Note that there are lingua franca languages other than English. See McGroarty (2006) for a state of the art on diverse languages. The argument made in this article for the implications of LFE for language acquisition may apply to those lingua franca languages as well.

${ }^{5}$ Though the construct of NS has been contested, I retain this term here for purposes of comparison with multilingual speakers. I am aware that many multilingual speakers will claim NS status in English.

${ }^{6}$ House (2003) argued that LFE is indeed a fullfledged language, not a pidgin variety or register for special purposes: "ELF is neither a language for specific purposes nor a pidgin, because it is not a restricted code, but a language showing full linguistic and functional range" (p. 557). Note also that there are different orientations to LFE. Some scholars are on the quest to define LFE according to an identifiable grammatical and phonological system (see Jenkins, 2006; Seidlhofer, 2004)-see note 7. This article is informed by the alternate school that focuses on the pragmatic features that enable LFE communication (see House, 2003; Meierkord, 2004).

${ }^{7}$ There is an attempt by some LFE scholars to identify the common aspects of phonological and grammatical form in LFE (see Jenkins, 2006; Seidlhofer, 2004) for pedagogical purposes. However, it is debated as to whether these items constitute the finite and invariable rules of LFE that might constitute a system or whether they are simply a list of typical and exemplary features. From this perspective, it is also premature to say if LFE is teachable like other languages in a product-oriented and formalistic manner.

${ }^{8}$ We have to distinguish the use of English in contact situations by multilinguals from interactions of second language speakers of English with speakers of a traditional national variety in a context where the frame of reference is a specific community (i.e., interacting with a speaker of American English in the United States; interacting with a speaker of Indian English in India). It is possible that the host community will use its norms to judge the effectiveness of speech, but, even here, multilinguals will tend to negotiate more equally (i.e., Indians would step out of their Indian English to negotiate the outsider's variety).
${ }^{9}$ For a discussion of these competencies and their place in lingua franca communication, see Canagarajah (2006c).

${ }^{10}$ The fact that I base this description on scholarship from non-Western scholars and communities does not imply that such features of multilingual communication have not existed in contexts of language contact in the West. It is simply that mainstream scholars have not adequately focused on them. Non-Western scholarship helps us discover the multilingual practices in the West.

${ }^{11}$ For a theorization of communicative success as "seizing the moment and negotiating paradoxes," see Kramsch (2002, p. 25).

${ }^{12}$ Postcolonial scholars see even more sinister motives in the way these constructs helped Europe establish its dominance over the communities it colonized in the 19th century. They point out that constructs like linguistic identity and speech community were put to use in lands like India to categorize people for purposes of taxation, administrative convenience, and political control (Mohan, 1992). In a very subtle way, these constructs have begun to shape social reality there with damaging results. Khubchandani (1997) observed "until as recently as four or five decades ago, one's language group was not generally considered as a very important criterion for sharply distinguishing oneself from others.... Following Independence, language consciousness has grown, and loyalties based on language-identity have acquired political salience" (p. 92). We can imagine how exclusive categories of identification can lead to ethnic and linguistic sectarianism. Furthermore, people have started perceiving themselves according to singular identities, lost their heterogeneity, and initiated conflicts and rivalries with members of what they perceive as alien language communities.

${ }^{13}$ For a distinction between the social processes of modern and postmodern globalization, see Hall (1997) and Canagarajah (2005b).

${ }^{14}$ For a detailed discussion of the difficulties in rediscovering local knowledge of non-Western communities in our profession, see Canagarajah (2005b).

${ }^{15}$ House (2003) also believed that a model based on communities of practice would do justice to the realizations of LFE communication.

\section{REFERENCES}

Anderson, B. (1984). Imagined communities: Reflections on the origins and spread of nationalism. London: Verso.

Atkinson, D., Churchill, E., Nishino, T., \& Okada, H. (2007). Alignment and interaction in a sociocognitive approach in second language acquisition. Modern Language Journal, 91, 169-188.

Block, D. (2003). The social turn in second language acquisition. Washington, DC: Georgetown University Press.

Brutt-Griffler, J. (2002). World English: A study of its development. Clevedon, UK: Multilingual Matters. 
Canagarajah, A. S. (1999). Resisting linguistic imperialism in English teaching. Oxford: Oxford University Press.

Canagarajah, A. S. (2005a). Introduction. In A. S. Canagarajah (Ed.), Reclaiming the local in language policy and practice (pp. xiii-xxx). Mahwah, NJ: Lawrence Erlbaum.

Canagarajah, A. S. (2005b). Reconstructing local knowledge, reconfiguring language studies. In A. S. Canagarajah (Ed.), Reclaiming the local in language policy and practice (pp. 3-24). Mahwah, NJ: Lawrence Erlbaum.

Canagarajah, A. S. (2006a). Toward a writing pedagogy of shuttling between languages: Learning from multilingual writers. College English, 68, 589-604.

Canagarajah, A. S. (2006b). Negotiating the local in English as a lingua franca. Annual Review of Applied Linguistics, 26, 197-218.

Canagarajah, A. S. (2006c). Changing communicative needs, revised assessment objectives: Testing English as an International Language. Language Assessment Quarterly, 3, 229-242.

Cook, V. (1999). Going beyond the native speaker in language teaching. TESOL Quarterly, 33, 185-209.

de Souza, L. M. (2002). A case among cases, a world among worlds: The ecology of writing among the Kashinawa in Brazil. Journal of Language, Identity, and Education, 1, 261-278.

Dorian, N. (2004). Minority and endangered languages. In T. K. Bhatia \& W. C. Ritchie (Eds.), The handbook of bilingualism (pp. 437-459). Oxford: Blackwell.

Firth, A. (1996). The discursive accomplishment of normality. On "lingua franca" English and conversation analysis. Journal of Pragmatics, 26, 237259.

Firth, A., \& Wagner, J. (1997). On discourse, communication, and (some) fundamental concepts in SLA research. Modern Language Journal, 81, 285-300.

Gass, S. (1998). Apples and oranges: Or, why apples are not orange and don't need to be. Modern Language Journal, 82, 83-90.

Giles, H. (Ed). (1984). The dynamics of speech accommodation [Special Issue]. International Journal of the Sociology of Language, 46.

Graddol, D. (1999). The decline of the native speaker. AILA Review, 13, 57-68.

Gramkow Anderson, K. (1993). Lingua franca discourse: An investigation of the use of English in an international business context. Unpublished master's thesis, Aalborg University, Aalborg, Denmark.

Hall, S. (1997). The local and the global: Globalization and ethnicity. In A. D. King (Ed.), Culture, globalization, and the world system (pp. 19-40). Minneapolis: University of Minnesota Press.

Haugen, E. (1972). The ecology of language. In A. Dil (Ed). Stanford, CA: Stanford University Press.

Higgins, C. (2003). "Ownership" of English in the outer circle: An alternative to the NS/NNS dichotomy. TESOL Quarterly, 34, 615-644.

Hopper, P. (1987). Emergent grammar. Berkeley Linguistics Society, 13, 139-157.
Hornberger, N. H. (Ed.). (2003). Continua of biliteracy: An ecological framework for educational policy, research, and practice. Clevedon, UK: Multilingual Matters.

House, J. (2003). English as a lingua franca: A threat to multilingualism? Journal of Sociolinguistics, 7, 556578.

Jenkins, J. (2006). Current perspectives on teaching World Englishes and English as a lingua franca. TESOL Quarterly, 40, 157-181.

Kasper, G. (1997). A stands for acquisition. Modern Language Journal, 81, 307-312.

Khubchandani, L. M. (1997). Revisualizing boundaries: A plurilingual ethos. New Delhi, India: Sage.

Kramsch, C. (2002). Introduction: How can we tell the dancer from the dance? In C. Kramsch. (Ed.), Language acquisition and language socialization: Ecological perspectives (pp. 1-30). London: Continuum.

Lantolf, J. P., \& Thorne, S. F. (2006). Sociocultural theory and the sociogenesis of second language development. New York: Oxford University Press.

Larsen-Freeman, D. (2002). Language acquisition and language use from a chaos/complexity theory perspective. In C. Kramsch. (Ed.), Language acquisition and language socialization: Ecological perspectives (pp. 33-46). London: Continuum.

Lave, J., \& Wenger, E. (1991). Situated learning: Legitimate peripheral participation. Cambridge: Cambridge University Press.

Long, M. H. (1997). Construct validity in SLA research. Modern Language Journal, 81, 318-323.

Makoni, S. (2002). From misinvention to disinvention: An approach to multilingualism. In G. Smitherman, A. Spear, \& A. Ball. (Eds.), Black linguistics: Language, society and politics in Africa and the Americas (pp. 132-153). London: Routledge.

May, S. (2001). Language and minority rights: Ethnicity, nationalism, and the politics of language. London: Longman.

McLaughlin, F., \& Sall, T. S. (2001). The give and take of fieldwork: Noun classes and other concerns in Fatick, Senegal. In P. A. Newman \& M. Ratiff. (Eds.), Linguistic fieldwork (pp. 189-210). Cambridge: Cambridge University Press.

McGroarty, M. (Ed.). (2006). Lingua franca languages [Special Issue]. Annual Review of Applied Linguistics, 26.

Meierkord, C. (2004). Syntactic variation in interactions across international Englishes. English World-Wide, $25,109-132$.

Mohan, K. (1992). Constructing religion and caste: Manipulating identities. Social Science Research Journal, 1, 1-12.

Norton, B. (2000). Identity and language learning: Gender, ethnicity, and educational change. Harlow, UK: Pearson.

Planken, B. (2005). Managing rapport in lingua franca sales negotiations: A comparison of professional and aspiring negotiators. English for Specific Purposes, 24, 381-400. 
Pratt, M. L. (1987). Linguistic utopias. In N. Fabb, D. Attridge, A. Durant, \& C. MacCabe (Eds.), The linguistics of writing: Arguments between language and literature (pp. 48-66). Manchester, UK: Manchester University Press.

Pratt, M. L. (1991). Arts of the contact zone. Profession, 91, 33-40.

Rampton, B. (1990). Displacing the Native Speaker: Expertise, affiliation, and inheritance. ELT Journal, 44, 97-101.

Rampton, B. (1997). Second language research in late modernity: A response to Firth and Wagner. Modern Language Journal, 81, 329-333.

Ruiz, R. (1984). Orientations to language planning. NABE Journal, 8, 15-34.
Sampson, H., \& Zhao, M. (2003). Multilingual crews: Communication and the operation of ships. World Englishes, 22, 31-43.

Seidlhofer, B. (2004). Research perspectives on teaching English as a lingua franca. $A n$ nual Review of Applied Linguistics, 24, 209239.

Singh, R. (Ed.). (1998). The native speaker: Multilingual perspectives. New Delhi, India: Sage.

Wenger, E. (1998). Communities of practice: Learning, meaning, and identity. Cambridge: Cambridge University Press.

Zuengler, J., \& Miller, E. R. (2006). Cognitive and sociocultural perspectives: Two parallel SLA worlds? TESOL Quarterly, 40, 35-58. 